\title{
Unsuccessful surgical excision of optic nerve drusen
}

\author{
Magdalena Pfriem • Hans Hoerauf
}

Received: 14 December 2010/Revised: 1 April 2011 / Accepted: 19 April 2011 /Published online: 3 June 2011

(C) The Author(s) 2011. This article is published with open access at SpringerLink.com

\begin{abstract}
Background To report the case of failed surgical excision of optic nerve drusen (OND).

Methods Case report. A 53-year-old woman presented with bilateral OND leading to progressive field defects and LE light perception and RE 20/25 vision. A vitrectomy was performed on the legally-blind left eye to test the resectability of OND.

Results The removal of superficial OND failed because the singular-appearing superficial OND presented as one large mass with multiple excrescences on its surface. Retinal vessels passed through it, which made an excision impossible. Conclusion OND cannot always be removed surgically, due to their variable consistency and hardness.
\end{abstract}

Keywords Optic nerve drusen · Vitrectomy · Visual field . Surgical technique

\section{Introduction}

Optic nerve drusen (OND) are acellular, calcific deposits occurring in 3.4 to $24 \%$ of the population [1]. Changes in axoplasmatic transport and the axonal degeneration of optic nerve fibres are suspected of being involved in OND formation [2, 3]. Most patients are subjectively asymptomatic, but visual acuity, preceded by severe visual field defects, is severely impaired in a small number of patients

Presentation at a conference: Retinologische Gesellschaft Freiburg, Germany June 2005

M. Pfriem $(\bowtie) \cdot$ H. Hoerauf

Department of Ophthalmology, Medical University of Goettingen,

37099 Goettingen, Germany

e-mail: mpfriem@yahoo.de
[1]. There is still no definitively effective treatment of OND [1]. The successful surgical removal of superficial OND was recently reported [4]. In contrast, and to demonstrate the heterogenicity of cases, we present a patient whose surgical excision of OND failed.

\section{Case report}

A 53-year-old woman presented with progressive visual field defects in the right eye, and long-standing visual loss in the left eye. Initial examination revealed best-corrected visual acuity of 20/25 RE, light perception LE, a severely constricted visual field in the $\mathrm{RE}<10^{\circ}$ and an unremarkable slit-lamp examination OU. Dilated ophthalmoscopic examination revealed bilateral superficial OND, which appeared as multilobular, yellowish-white, sharp-edged nodules and anomalous arterial and venous branchings, increased tortuosity, and cilioretinal arteries (Fig. 1).

To test the resectability of OND and to gain experience, we decided to perform a vitrectomy with surgical excision on the blind left eye before considering surgical manipulations on the right eye with the risk of losing the remaining small central visual field, leading to complete blindness. After informing the patient about the procedure's experimental character and obtaining informed consent, we conducted a standard three-port pars plana vitrectomy. We then performed a small retinotomy at the nasal edge of the optic nerve. Despite numerous efforts using different vitreoretinal surgical instruments such as blades, forceps and fine scissors, removal of the superficial OND failed. During surgery, we became aware that the small, multiple, and singular-appearing superficial ONDs presented as the multilobulated surface of one calcified crust covering the complete optic nerve head, with central retinal vessels 
Fig. 1 Fundus photography of right eye (a), with progressive field defects and left eye (b), functionally blind, showing multiple superficial OND covering the entire disc and abnormal branching of retinal vessels
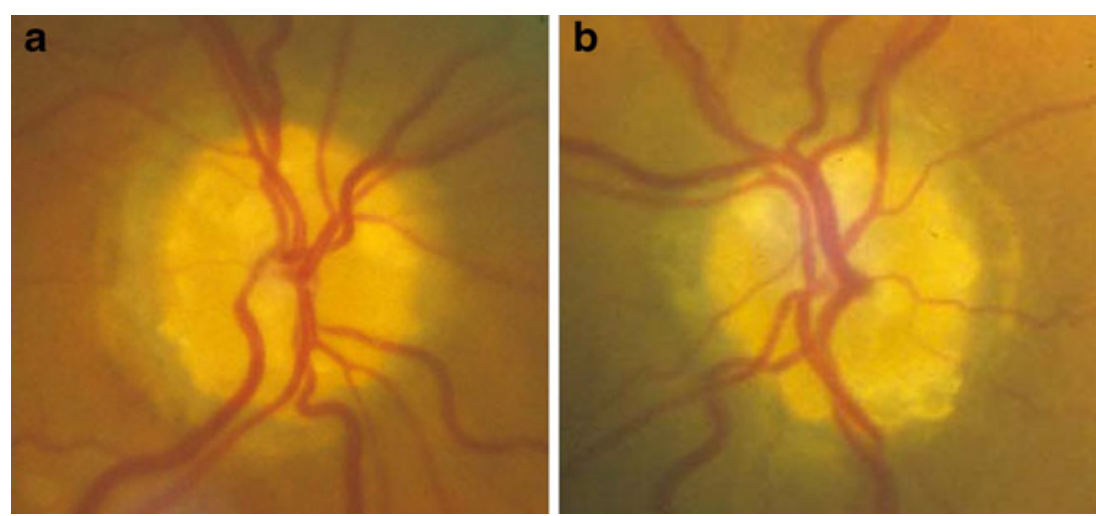

passing through (Fig. 2). It was impossible to split the extremely hard crust into small pieces and to remove considerable parts of it. Moreover, radial neurotomy as described in the literature was impossible due to the hardness of the OND material [5]. Manipulations were stopped and the eye was filled with air.

\section{Discussion}

Axonal degeneration, ischaemia within the optic nerve head, and OND pressing down on prelaminar nerve fibres have been proposed as pathogenetic mechanisms in OND disease $[1,3]$.

A recent publication reported on a good functional outcome after vitrectomy and radial optic neurotomy (RON) for OND, suggesting that RON leads to mechanical decompression with subsequent optic nerve fibre recovery, and that the excision of prelaminar deposits is an effective strategy in OND disease [5]. However, our case shows that

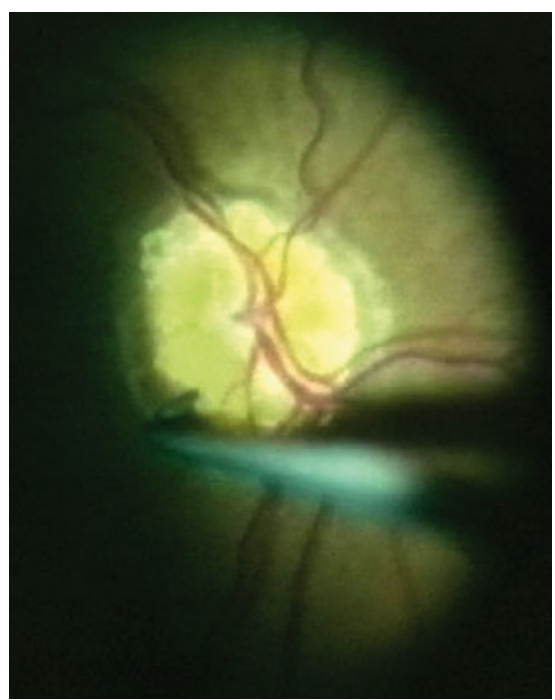

Fig. 2 Intra-operative image of left eye showing ineffective manipulations at the nasal edge of the optic nerve surgical removal or decompression is not possible in all cases; our patient was considerably older than theirs.

A histological study in 1981 demonstrated that the number and size of superficial and buried OND vary greatly [3]. Furthermore, their size and density increase with age, due to continuous calcium apposition [1]. Rather than revealing multiple small deposits, our patient's OND formed a single, large solitary mass with central retinal arteries and veins passing through it. Our observation concurs with a report by Kapur et al., who reported on the incomplete surgical excision and bisection of OND in a 59-year-old patient "because retinal vessels were observed to be emanating from the mass" [4]. In contrast to their experience, in our patient the excision or even splitting of this large, extremely hard OND crust without damaging adjacent structures was not feasible.

Thorough fundus biomicroscopy may help identify candidates for surgery. Other diagnostic methods such as autofluorescence fundus photography, ultrasound B and optical coherence tomography are available, but they do not enable us to assess the size, formation and hardness of OND.

In conclusion, this case report demonstrates that OND removal is not feasible in every case. Their variability in consistency and hardness makes them unpredictable, thus we cannot know in advance which patient is an appropriate candidate for surgery. Patient age seems to be an important factor associated with the composition of OND, but also earlier surgery could perhaps yield superior results. In accordance with Kapur et al., we propose that other surgical or ablating laser techniques must be developed to achieve safe removal of extremely hard material, while avoiding damage to adjacent, functionally important and sensitive structures such as optic nerve fibres and retinal vessels [4].

\section{Conflict of interest None}

Open Access This article is distributed under the terms of the Creative Commons Attribution Noncommercial License which permits any noncommercial use, distribution, and reproduction in any medium, provided the original author(s) and source are credited. 


\section{References}

1. Auw-Haedrich C, Staubach F, Witschel H (2002) Optic disk drusen. Surv Ophthalmol 47:515-532

2. Spencer WH (1978) XXXIV Edward Jackson Memorial Lecture: drusen of the optic disc and aberrant axoplasmatic transport. Am J Ophthalmol 85:21-38
3. Tso MO (1981) Pathology and pathogenesis of drusen of the optic nervehead. Ophthalmology 88:1066-1080

4. Kapur R, Pulido JS, Abraham JL, Sharma M, Buerk B, Edward DP (2008) Histologic findings after surgical excision of optic nerve head drusen. Retina 28:143-146

5. Haritoglou C, Priglinger SG, Grueterich M, Kampik A, Kriegelstein GK (2005) Radial optic neurotomy for the treatment of acute functional impairment associated with optic nerve drusen. $\mathrm{Br} \mathrm{J}$ Ophthalmol 89:779-780 